\title{
IONTOPHORESIS-ENHANCED CUTANEOUS ABSORPTION OF 5-AMINOLEVULINIC ACID SHORTENS THE INCUBATION PERIOD IN PHOTODYNAMIC THERAPY
}

\author{
Tokuya Omi ${ }^{1,2}$, Makio Akimoto ${ }^{3}$, Michio Miyazaki ${ }^{3}$, Seiji Kawana ${ }^{2}$ \\ 1) Department of Dermatology, Queen's Square Medical Center \\ 2) Department of Dermatology, Nippon Medical School \\ 3) Faculty of Engineering, Kanto Gakuin University
}

\begin{abstract}
The combination of light and chemicals to treat skin diseases is widely practiced in the field of dermatology, and has led to the concept of photodynamic therapy (PDT) in recent years. PDT is a promising modality for managing various cancers and nonmalignant diseases, and is based on the combination of selective localization of a photosensitizer in the target tissue cells and the subsequent illumination of the lesions with visible light resulting in photodamage and cell death. PDT with topical 5-aminolevulinic acid (ALA) has been increasingly employed to treat skin cancers, however the dosing of ALA has never been optimized. Using iontophoresis, we have developed a rapid and quantifiable system for the topical delivery of ALA, and we measured the subsequent fluorescence of protoporphyrin IX (PpIX) and phototoxicity. Iontophoresis is a rapid method for the delivery of ALA into skin lesions, making prolonged incubation of the ALA unnecessary and potentially leading to more efficient PDT.
\end{abstract}

Key Words: iontophoresis, photodynamic therapy (PDT), 5-aminolevulinic acid (5-ALA), skin disease

\section{Introduction}

In recent years, the application of optical technology in clinical medicine has been thriving. Many systems based on optical technology have been developed and are widely used in clinical settings; e.g., lasers have produced revolutionary results in surgery and medicine, most cases of chemical tests on specimens are now conducted using optical techniques, and automated optical-based assays have been developed, resulting in tremendous advances in patient diagnostics and medical examinations. Furthermore, a minimally-invasive treatment method has been recently developed, in which certain drugs delivered to the body are activated by specific wavelengths of light to exert their effects,

Addressee for Correspondence:

Tokuya Omi M D, PhD

Department of Dermatology, Queen's Square Medical Center

2-3-5 Minatomirai, Yokohama 220-6208 Japan

Email: t.omi@queens-sq.or.jp

TEL: $045-682-4112$

FAX: 045-682-4111 and the therapeutic effects of this modality have drawn close attention. The modality is referred to as photodynamic therapy (PDT), and is based on the combined use of photosensitizers and photoradiation ${ }^{1-4)}$. In this method (shown schematically in Fig. 1) a photosensitizer is administered with affinity for the tumor cells, followed by light irradiation which results in the selective disruption of only the tumor cells in the body due to a phototoxic reaction involving the creation of reactive oxygen species (ROS). Since PDT is less invasive than surgical therapy, it can be used in patients with serious complications and also in elderly patients, and it is being increasingly applied in many clinical fields, especially dermatology and ophthalmology 5,6). In the field of dermatology, it is widely recognized that PDT, in which an excitation light is applied to externally applied 5-aminolevulinic acid (5-ALA), a porphyrin precursor, is effective for the treatment of superficial malignant tumors of the skin. However, since the percutaneous absorption of photosensitizers is extremely

Manuscript received: April 2009

Accepted for publication: August 2009 


\section{ORIGINAL ARTICLES}

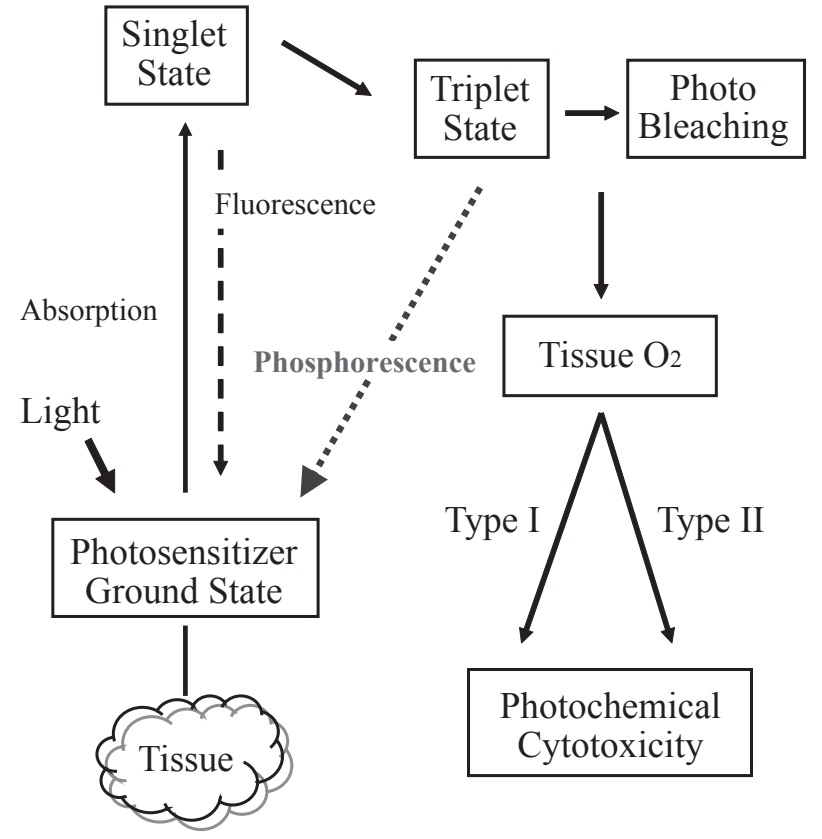

Fig. 1: The principles of PDT

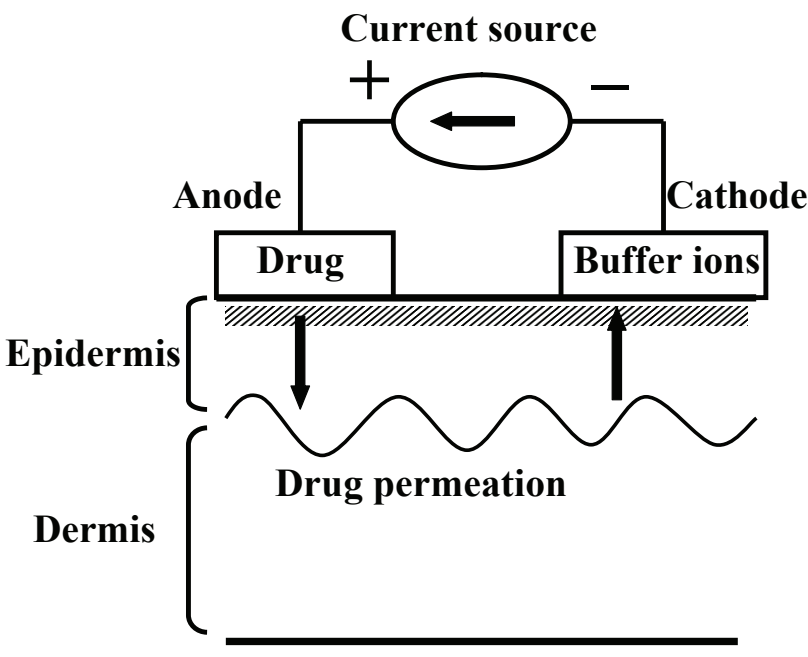

Fig. 2: The principles of iontophoresis low, treatment using these substances requires a long time because of extended incubation periods, making it difficult to establish this method as a standard treatment modality and to apply it efficiently on an outpatient basis. In this study, PDT was optimized by introducing the photosensitizer into the skin within a short time by iontophoresis, which involves the application of a microelectric current to the skin to increase the percutaneous absorption of ionic drugs (shown schematically in Fig. 2). 5-ALA has an extremely low percutaneous absorbency, and iontophoresis could dramatically enhance its absorption, in addition to other known methods such as liposomal ALA. ${ }^{7,8)}$ The results of this study as reported herein confirmed that iontophoresis did indeed enhance the percutaneous absorption of the photosensitizer within a short time, which dramatically reduced the time required for treatment.

\section{Subjects and experimental methods}

The target lesions for the treatment were 5 untreated solar keratosis lesions and 2 untreated Bowen's disease lesions in 5 patients (male 2, female 3, age 49-94 years, average 79.6 years). All lesions were diagnosed after lesional punch biopsy.

The TransIon TH system (ICI Cosmetics Japan,
Tokyo, Japan) was used for iontophoresis in this study 9,10). As a functional electrode, an iontophoresis pad was used, and an ECG pad was utilized as the opposite pole plate. In order to avoid polarization, which may occur when an electric current is applied, a direct current pulse to the cathode was adopted as the waveform of the drive current source. The variable range of voltage was 0 to $100 \mathrm{~V}$, and the variable range of current was 0 to $10 \mathrm{~mA}$. In order to avoid a decrease in the efficiency of drug delivery due to polarization at the time of application of the electric field to the skin, the pulse frequency could be controlled in 6 stages within the range of 2 to $50 \mathrm{kHz}$, and the duty ratio could also be set. In other words, the voltage was applied by the current source voltage for a constant period during which time, the charge was integrated by the skin. The charge retained in the skin was then released during the resting phase. It is thought that decreases in absorption efficiency due to polarization can be prevented by the repetition of such pulses.

The photosensitizer 5-ALA (ALA-HCI, COSMO BIO Co., Ltd. Tokyo, Japan) was dissolved in distilled water to prepare a $20 \%$ solution, through which a functional electrode pad was permeated. An ECG electrode was used as the opposite-pole electrode. The current volume during the iontophoresis was controlled by a square measure of the functional electrode 


\section{ORIGINAL ARTICLES}

to $0.25-0.50 \mathrm{~mA} / \mathrm{cm}^{2}$. The pulse frequency was $50 \mathrm{kHz}$, the duty ratio was $30 \%$, and the delivery time was 10 minutes. After delivery of 5-ALA, the delivery site was washed gently with water, and shielded from light. One hour later, the amount of protoporphyrin IX (PpIX) produced was measured with a fluorescence spectrometer. The amount was calculated from the ratio of the fluorescence at $500 \mathrm{~nm}$ to that at $636 \mathrm{~nm}$. The photoradiation was performed using a PDT EDL-1 system (Hamamatsu Photonics KK, Hamamatsu, Japan), which is an excimer dye laser and emits 630 $\mathrm{nm}$ in pulsed mode. The photoradiation doses used for the PDT were $150 \mathrm{~J} / \mathrm{cm}^{2}$ and $300 \mathrm{~J} / \mathrm{cm}^{2}$ for solar keratosis and Bowen's disease, respectively.

\section{Results}

Following the application of 5-ALA by iontophoresis, all lesions showed production of PpIX. Histological examinations of the skin were performed 1-2 weeks after the end of the PDT for examination of the presence/absence of residual tumor cells. These examinations confirmed the complete disappearance of tumor cells. Fig. 3 shows an example of the therapeutic effect.

\section{Discussion}

\section{Principles of PDT}

It has long been recognized that substances which are sensitive to light and placed on or in living tissue can be activated by light to manifest cytotoxicity. With the development of photosensitizers with higher affinities for tumor tissues compared to normal tissues, the range of clinical applications of those substances has been extended. PDT (shown schematically in Fig. 1) is a therapeutic modality in which a photochemical reaction is induced in target cells by administration of a photosensitizer with a high affinity for the target cells and subsequent irradiation of the tissue with light of an appropriate wavelength to obtain selective destruction of the target 11,12). In dermatology, PDT is frequently used to treat acne, hair removal and superficial skin cancers, such as solar keratosis and Bowen's disease $5,6,13,14)$. Photosensitizers are administered to the body by injection, orally or topically. When the photosensitizer is in its ground state in the target tissue and is irradiated with light of a specific wavelength, the energy level of the substance is raised the excited singlet state. Part of the substance returns to the ground state

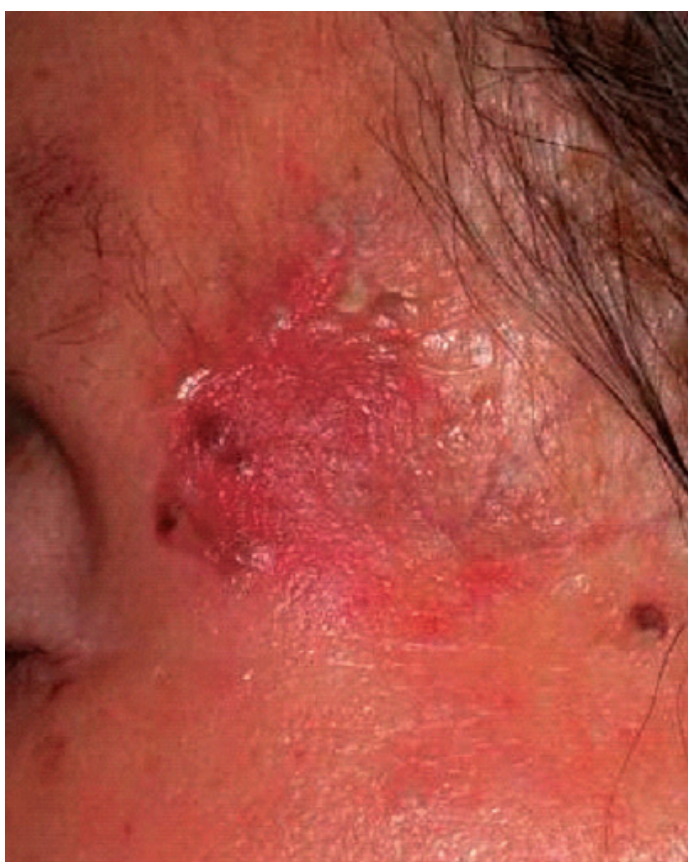

Before

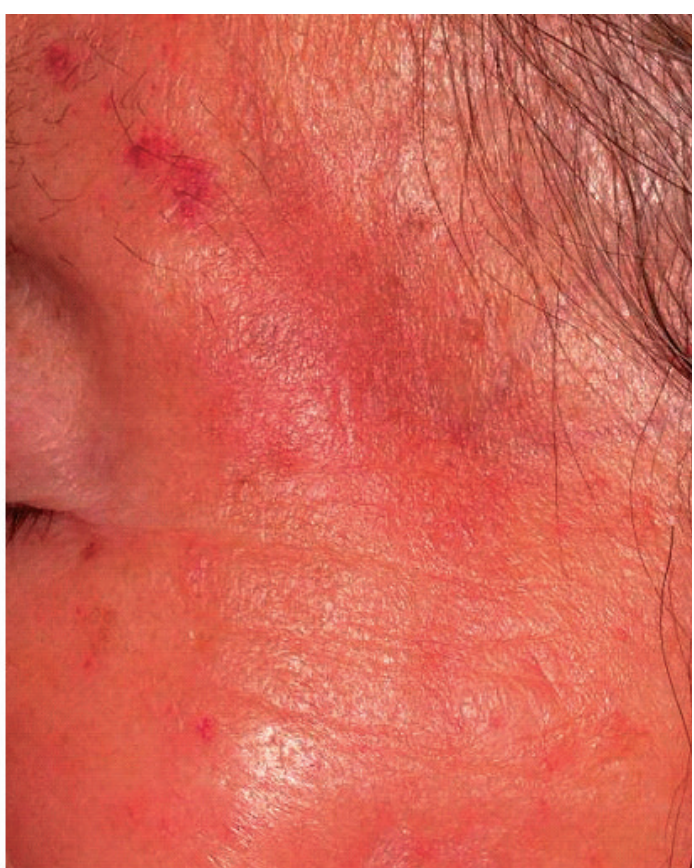

After

Fig. 3: The efficacy of 5-ALA PDT plus iontophoresis to treat solar keratosis in a 74 year old female. One session of PDT therapy was performed and no atypical cells were observed 2 weeks after the therapy. 


\section{ORIGINAL ARTICLES}

while emitting fluorescence, whereas the remaining part is raised to the triplet state, ultimately returning to the ground state while emitting phosphorescence. When a photosensitizer is excited by light two types of reactions are induced: a type-I reaction, which is the electron transport process and a type-II reaction which is the energy transport process. The free radicals produced by the type-I reaction react with dissolved oxygen in the tissues to produce various oxidized substances, known collectively as reactive oxygen species (ROS) which elicit a free-radical chain reaction resulting in highly-localized oxidative stress. In the type-II reaction, the energy level of the photosensitizer in the target cells is raised to the excited singlet state by the incident photon energy from the activating light source. At that time, part of the substance returns to the ground state while emitting fluorescence, while the majority of the substance is changed to the triplet state. The triplet state returns partly to the ground state while emitting phosphorescence. The remaining excess energy is absorbed by the oxygen in solution in the target tissues, creating singlet oxygen through excitation, which causes degenerative necrosis of the target cells. The majority of the therapeutic effect of PDT is the result of the type-II reaction, bolstered by the Type-I reaction oxidative stress, but the effect varies with the type of photosensitizer used. It is known that the majority of photosensitizers are integrated into the intracellular mitochondria. Integration of these substances into the vascular endothelia of tumor tissues and damage to the endothelial cells during PDT have

Table 1. PDT drugs and excitation wavelengths

\begin{tabular}{lc}
\hline Photosensitizer & $\begin{array}{c}\text { Wavelength } \\
(\mathrm{nm})\end{array}$ \\
\hline Hematoporphyrin derivative (HpD) & 630 \\
\hline 5-aminolevulinic acid (5-ALA) & 632 \\
\hline Tin etiopurpurin (SnET2) & 660 \\
\hline $\begin{array}{l}\text { N-aspartyl-chlorin e6 (NPe6) } \\
\text { Chloroaluminium phthalocyanine (A } \ell \text { PcS) }\end{array}$ & 675 \\
\hline $\begin{array}{l}\text { Benzoporphyrin derivative-monoacid ring A } \\
\text { (BPD-MA) }\end{array}$ & 690 \\
\hline \begin{tabular}{l} 
Lutetium texaphyrin (Lu-Tex) \\
\hline
\end{tabular}
\end{tabular}

also been suggested ${ }^{11,12)}$.

For skin cancer therapy, PDT utilizes the specific property of integration of a photosensitizer with tumor affinity into the tumor tissue and newly formed capillaries, with the production of active oxygen species by the fluorescence emission or light excitation. There are numerous compounds with photosensitizing properties, however, the number of compounds that can be administered to a patient is limited by the consideration of safety. Table $\mathbf{1}$ shows typical examples of photosensitizers with high tumor affinities. The development of photosensitizers with long excitation wavelengths has also begun to thrive because of their property of good penetration into biological tissue compared with shorter wavelengths. The only photosensitizer used for PDT 15,16) that is currently approved for use in Japan, however, is a derivative of hematoporphyrin ( $\mathrm{HpD}$, Photofrin $\left.{ }^{\circledR}\right)$, which is a porphyrin compound. When the photosensitizer is applied to normal tissues, it integrates at a high concentration into the skin, intestinal tract, liver, kidney and lung tissues in the early stage of administration, followed by rapid metabolism within 48 hours of its application. When it is taken up by tumor tissues, on the other hand, a relative difference is found in the tissue concentrations, because the substance integrates at a higher concentration into the tumor tissue than into the normal tissue and is moreover metabolized rather slowly in the tumor tissue. PDT is based on this difference in the metabolic rate and therefore the relatively higher concentration of the photosensitizer in tumor compared with normal tissues.

Since the target lesions in the field of dermatology frequently include exposed sites, such as the face, arms and hands, 5-ALA is used because it has a short metabolic half life. It is dissolved in a base prior to its application. When 5-ALA is administered to the body, it enhances the biosynthesis of endogenous PpIX. PpIX is eventually incorporated into the heme cycle but this reaction is relatively slow. By 4-6 hours after the administration of 5-ALA, the peak PpIX concentration is obtained. At this time point, PDT is based on the properties of PpIX as a photosensitizer. The use of 5ALA is expected to yield excellent treatment results, replacing surgical excision, because 5-ALA is dissolved in a base prior to its application on the skin. 5-ALA, an amino acid with a molecular weight of 131 , is a substance that also exists in the living body; it is highly hydrophilic, stable under acidic conditions, and dissolves under alkaline conditions. 5-ALA has no photosensitivity by itself, but when excessive 5-ALA is applied exogenously onto the skin, the PpIX formed is 
volumetrically incorporated into the underlying skin cells. PpIX is excited by certain wavelengths of light, and when it returns to the ground state through the triplet oxygen state, singlet oxygen is produced in the cells with ensuing cytotoxicity by the active oxygen of these states. 5-ALA has a maximum absorption peak at an excitation wavelength of $415 \mathrm{~nm}$ in the Soret band, but there are also peaks at 510, 545, 580 and $630 \mathrm{~nm}$. In general, light with a wavelength mainly around 410 $\mathrm{nm}$ is used for making the diagnosis, whereas light at a wavelength of $630 \mathrm{~nm}$ is utilized for treatment, based on the lower extinction coefficient of that wavelength, and hence its deeper penetration into living tissue than the shorter visible light wavelengths. PpIX emits fluorescence and shows color development when it is irradiated with ultraviolet light. Approximately 1 hour after 5-ALA is applied to the lesions, it starts emitting fluorescence in the visible red waveband under UV radiation, which peaks at about 4-6 hours after application, followed by gradual decay. Under these conditions, the photosensitizer needs to be applied topically to the lesions for a 4-6 hour incubation period before photoactivation, and treatment should be conducted in an environment shielded from ambient light. It may be problematic for patients to perform the above-mentioned procedure by themselves, and the patient's condition and environment sometimes make it very difficult. Further, 5-ALA is a very unstable substance and is easily oxidized under normal ambient room conditions. 5-ALA PDT is therefore best performed in a dedicated treatment facility.

Liposomal ALA 13) is one method to solve this problem, since it requires only 1 hour for penetration and is stable even at room temperature. However, following the results of the present study, iontophoresis may represent another useful method for enhancing the percutaneous absorption of ALA.

\section{Iontophoresis for enhancement of percuta- neous absorption}

The skin is composed of three layers, the epidermis, the dermis and the subcutaneous tissue. The outermost layer of the skin is a hydrophobic layer, which is called the stratum corneum. Underneath that is the highly hydrophilic living epidermal layer. Since squamous cells are solidified with lipids in the stratum corneum, water-soluble drugs fail to diffuse through this layer, and substances are, in fact, repelled by the surface of this layer. Highly hydrophobic drugs can permeate the stratum corneum, however, the delivery of drugs to the living epidermal layer becomes difficult because that layer is hydrophilic. Moreover, many enzymes are also distributed in the living epidermal layer, and foreign bodies invading from the outside are metabolized and dissolved in that layer. In other words, the skin functions as a obstacle to prevent foreign bodies from the external environment from invading the body. Towards that end, the stratum corneum acts as a physical barrier and the living epidermal layer acts as a chemical barrier. Iontophoresis utilizes an electric driving force as a method for the physicochemical improvement of the percutaneous absorption of drugs 17,18 . With iontophoresis, an electric field is applied to the skin to enhance the percutaneous absorption of ionic drugs. This method of drug delivery is called iontophoresis therapy or ionic delivery. The principle of iontophoresis is shown in Fig. 2: percutaneous absorption is enhanced by an electrical repulsion after configuration of a drug to the side of the electrode with the same charge as the ionized drug. In regard to neutral drugs without charge, the absorption is enhanced by water convection produced by the application of an electric current to the skin. Drug absorption at the time of application of iontophoresis is expressed as the sum of the term of passive diffusion and the iontophoresis-induced electrical repulsive force and convection. There are two modes by which convection is produced; one where convection occurs by movement of hydrating ions and the other where convection occurs by the development of osmotic pressure associated with the application of electricity. With regard to the pathway of drug absorption by iontophoresis, transappendages are the major pathway, because an electric current at the time of application of electricity is favorably conducted through the transappendages, including the hair follicles and other skin structures, e.g. sebaceous glands, sweat glands and so on, which have a lower electrical resistance and therefore offer better electrical conductivity compared with surrounding tissues. There are high expectations in regard to the application of iontophoresis for treating refractory skin diseases in the elderly, because the use of iontophoresis is associated with only slight pain and discomfort.

5-ALA is hydrophilic material and is very difficult to penetrate into the skin. 5-ALA with saline needs more than 5 to 6 hours to pass the epidermis ${ }^{19}$ ).

In this study, only 10 minutes of application of 5ALA and a 1 hour follow-up showed the a positive PpIX reaction within the target. With iontophoresis, it took one hour for the entire treatment, ranging from the delivery of 5-ALA to the end of the treatment. Thus, the time needed for treatment can be drastically shortened with iontophoresis. Liposome encapsulated 


\section{ORIGINAL ARTICLES}

5-ALA needs 1 hour application but it should be sprayed at least 12 times with 5 minutes intervals 20)

Drug absorption at the time of application of iontophoresis can be expressed as the sum of the term of the usual passive diffusion and the iontophoresis forces: electrical repulsive force and convection, as shown in the following formula:

Jtotal $=$ Jpassive + Jrepulsion + Jconvective flow

There are two types of convection development; convection due to hydrated ionic movement and convection due to osmotic pressure development associated with the use of electricity. Usually, the skin becomes negatively charged in the neutral area. It can be said that ions becoming positively charged are selectively absorbed, thereby probably leading to the development of a convective current from the anode to the cathode and the enhanced absorption of drugs by convection. These phenomena indicate that the absorption of even neutral drugs without charge can be enhanced by the water convection that develops after application of an electric current to the skin. In this sense, the fact that 5-ALA was percutaneously absorbed within only about 10 minutes seems to sufficiently reflect the effect of iontophoresis in enhancing the drug absorption.

The intensity of the light irradiating the skin shows decay both in absorption and in scatter. The decay of light that is actually observed shows sustained absorption and multiple scatter in the skin tissues, and the intensity shows mostly exponential decay. Accordingly, the effective decay coefficient $\mu$ eff is expressed by the following formula when a coordinate in the progression direction is plotted along $z$ :

$I(z)=I 0 \bullet \exp (-\mu e f f z)$

where $I(z)$ indicates the intensity of light at depth $z, I 0$ indicates the intensity of the irradiating light, meff indicates the tissue decay coefficient ( $\mu$ eff $=$ $\left(3 \mu a\left(\mu a+\mu s^{\prime}\right)^{1 / 2}\right), \mu a$ indicates the tissue absorption coefficient, and $\mu s^{\prime}$ indicates the tissue scatter coefficient. When the optical constants of the tissue at a

\section{References}

1: Fingar, VH and Henderson, BW: Drug and light dose dependence of photodynamic therapy: a study of tumor and normal tissue response, Photochem Photobiol 1987, 46, 837-841.

2: Daniel, MD and Hill, JS: A history of photodynamic therapy, Aust NZ J Surg 1991, 61, 340-348. wavelength of $633 \mathrm{~nm}\left(\mu_{a}=0.19 \mathrm{~mm}^{-1}, \mu^{\prime}=2.38 \mathrm{~mm}^{-}\right.$ 1) 21,22) are used, for instance, the intensity of light at a $1 \mathrm{~mm}$ depth from the tumor surface is estimated to show decay to approximately $1 / 3$. In actual PDT, the treatment site frequently varies and pulsed radiation is frequently employed. It is therefore difficult to determine the rigid optical constant of a tissue, but a high therapeutic effect would retrospectively indicate that the intensity of radiation for the PDT was adequate. PDT is a minimally- invasive treatment method, and can be performed in the elderly, in patients in poor general health, and in patients for whom surgery is expected to be difficult. It is excellent from the aesthetic aspect because it does not leave a scar or atypical pigmentation.

On the other hand, pain and a sensation of discomfort may be associated with the activation of the photosensitizer by the selected wavelength of light. The pain varies with the sites and the sizes of lesions and also the light source, but it is thought that the pain can mainly be attributed to the tissue damage caused by a secondary thermal effect accompanying the photoirradiation, and by the direct stimulation of nociceptive fibers by 5-ALA-mediated oxidative stress. Local anesthesia, such as a topical anesthetic cream, might therefore be necessary in some patients. From such a viewpoint, the delivery of 5-ALA within a short time by iontophoresis is also useful in terms of shortening the treatment time.

\section{Conclusions}

Along with the increase in the number of elderly patients, the number of patients with superficial malignant tumors of the skin also seems to be on the increase. Under these circumstances, the need for a minimally-invasive modality such as PDT increases concomitantly. Since 5-ALA, a photosensitizer with high tumor affinity, is absorbed percutaneously only with difficulty under normal circumstances, the effect of iontophoresis in enhancing the absorption within a short period of time is useful from the viewpoint of improving the quality of the PDT.

3: Dougherty, TJ: Photodynamic therapy, Photochem Photobiol 1993, 58, 859-900

4: Ochsner, M: Photodynamic therapy: the clinical perspective, Arzneim Forsch Drug Res 1997, 47, 1185-1194.

5: Kalka, K, Merk, H and Mukhtar, H: Photodynamic 
therapy in dermatology, J Am Acad Dermatol 2000, 42, 389-413.

6: Tse, DT, Kersten, RC and Anderson, RL: Hematoporphyrin derivative photoradiation therapy in managing nevoid basal-cell carcinoma syndrome, Arch Ophtalmol 1984, 102, 990-994.

7: Rhodes, LE, Tsoukas, MM, Anderson, RR and Kollias, N: Iontophoretic delivery of ALA provides a quantitative model for ALA pharmacokinetics and PpIX phototoxicity in human skin, J Invest Dermatol 1997, 108, 87-91.

8: Merclin, N, Bramer, $\mathrm{T}$ and Edsman, K: Iontophoretic delivery of 5-aminolevulinic acid and its methyl ester using a carbopol gel as vehicle, J Control Release 2004, 98, 57-65.

9: Akimoto, M and Omi, T: Development of high frequency direct current pulse-type iontophoresis and its clinical application, Aesthetic Dermatol 2006, $16,37-44$.

10: Akimoto, M, Kawahara, M, Matsumoto, M and Matsubayashi, H: Development of the Pulsed Direct Current Iontophoresis and Its Clinical Application, in Proc. Progress In Electromagnetics Research Symposium (PIERS 2006), pp 157-162, Cambridge, Massachusetts, USA (2006).

11: Wilson, BC and Patterson, MS: The physics of photodynamic therapy, Phys Med Biol 1986, 31, 327360.

12: Henderson, BW and Dougherty, TJ: How does photodynamic therapy work? Photochem Photobiol 1992, 55, 145-157.

13: Leeuw J, Vijlder HC, Bjerring P, Neumann H: Liposomes in dermatology today. J Eur Acad Dermatol Venereol 2009, 23, 505-516.

14: Yeung CK, Shek SY, Bjerring P, Yu CS, Kono T,
Chan HH: A comparative study of intense pulsed light alone and its combination with photodynamic therapy for the treatment of facial acne in Asian skin. Lasers Surg Med 2007, 39, 1-6.

15: Ash, DV and Brown, SB: New drugs and future developments in photodynamic therapy, Eur J Cancer 1993, 29, 1781-1783.

16: Levy, JG: Photosensitizers in photodynamic therapy, Semin Oncol 1994, 21, 4-10.

17: Cross, SE and Roberts, MS: Physical enhancement of transdermal drug application: Is delivery technology keeping up with pharmaceutical development? Current Drug Delivery 2004, 1, 81-92.

18: Kumar, R and Philip, A: Modified transdermal technologies: Breaking the barriers of drug permeation via the skin, Trop J Pharm Res 2007, 6, 633-644.

19: Pierre M, Tedesco A, Marchetti J, Bentley M: Stratum corneum lipids liposomes for the topical delivery of 5-aminolevulinic acid in photodynamic therapy of skin cancer: preparation and in vitro permeation study. BMC Dermatology 2001, 1, 5-10

20: Bjerring P, Christiansen K, Troilius A, Bekhor P, de Leeeuw J: Skin fluorescence controlled photodynamic photorejuvenation. Lasers Surg Med 2009, 41: 327-336

21: Graaff, R, Dassel, ACM, Koelink, MH, Mul, FFM, Aarnoudse, JG: Optical properties of human dermis in vitro and in vivo, Applied Optics 1993, 32, 435-447.

22: Simpson, CR, Kohl, M, Essenpreis, M and Cope, M: Near-infrared optical properties of ex vivo human skin and subcutaneous tissues measured using the Monte Carlo inversion technique, Phys Med Biol 1998, 43, 2465-2478. 